\title{
Onychodystrophy due to porokeratosis of Mibelli: a rare association
}

\author{
Manoj Pawar ${ }^{1 凶}$
}

\begin{abstract}
Porokeratosis is a specific keratinization disorder that manifests clinically as well-demarcated annular or linear keratotic plaques of various sizes and forms and with distinguished histology showing cornoid lamella, which is a column of closely packed parakeratotic cells extending through the stratum corneum. Nail changes secondary to porokeratotic lesions involving digits are quite uncommon and rarely reported in the porokeratosis of Mibelli (PM) subtype. Here we report the case of a young girl with a PM lesion over the hand along with nail dystrophy of the involved digit.
\end{abstract}

Keywords: porokeratosis of Mibelli, onychodystrophy, cornoid lamella

Received: 26 March 2017 | Returned for modification: 23 April 2017 | Accepted: 24 April 2017

\section{Introduction}

Porokeratosis is a rare keratinization disorder. Several clinical variants have been identified, including porokeratosis of Mibelli (PM), disseminated superficial porokeratosis of immunosuppression and childhood, disseminated superficial actinic porokeratosis (DSAP), linear and punctate forms, porokeratosis palmaris et plantaris disseminate, giant porokeratosis, and porokeratosis ptychotropica (1). PM consists of well-defined annular or scalloped plaques that are mostly unilateral. Very few cases of nail dystrophy due to porokeratosis have been reported, and these have mainly been seen with linear porokeartosis (2-4), whereas onychodystrophy with PM is rarely documented (5-9).

\section{Case report}

A 14-year-old girl presented with an annular plaque on the dorsum of the right hand with destruction of the nail-plate of the right ring finger. She had first noticed an asymptomatic smaller, ring-like skin lesion at the base of the fourth finger above the dorsum of the right hand 2 years earlier, which gradually increased in size to attain its present size, involving almost the entire fourth finger with its nail plate and third finger up to the

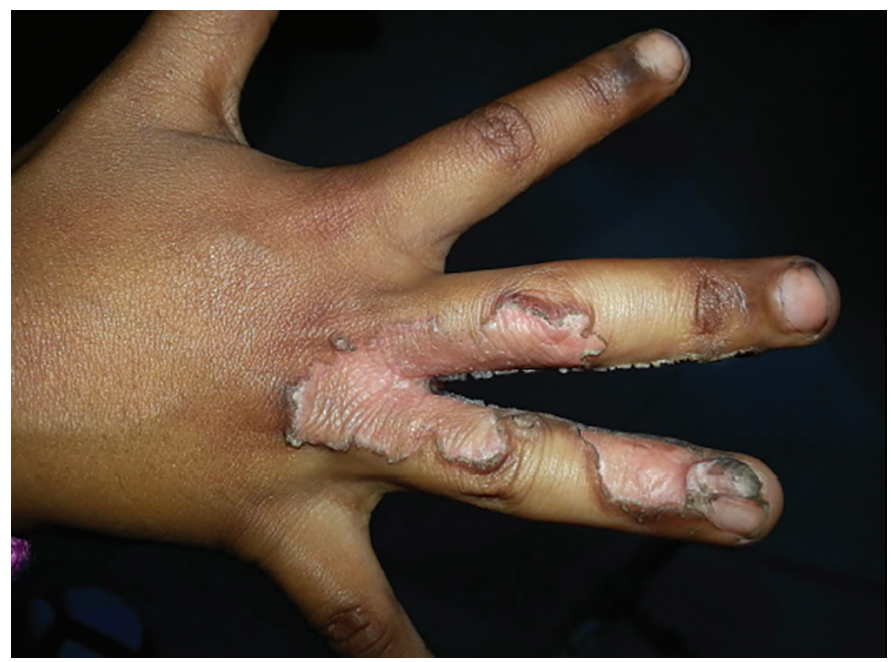

Figure 1 | Clinical photograph showing a well-defined, hyperkratotic plaque with central atrophy with nail dystrophy of the fourth finger of the right hand. proximal interphalangeal joint. She had no significant past history, family history, or history of using illicit drugs. Examination revealed a well-demarcated annular plaque with central atrophy bordered by a hyperkeratotic ridge at the base of the third and fourth digits over the dorsum of right hand accompanying nail dystrophy of the fourth digit (Figure 1). Histology of the lesion showed keratin-filled epidermal invagination with parakeratotic columns of keratinocytes denoting cornoid lamella. Beneath it, the epidermis displayed vacuolization compatible with the diagnosis of porokeratosis (Figure 2). A general and laboratory examination, and an X-ray of the hand did not reveal any abnormal findings. She had no evidence of immunosuppression or any systemic disease associated with porokeratosis. Microscopic examinations, and fungal and bacterial cultures of the nails were negative. Based on the typical clinical morphology and histology, the patient was diagnosed with PM and was prescribed a topical keratolytic ( $12 \%$ salicylic acid) and topical retinoids.

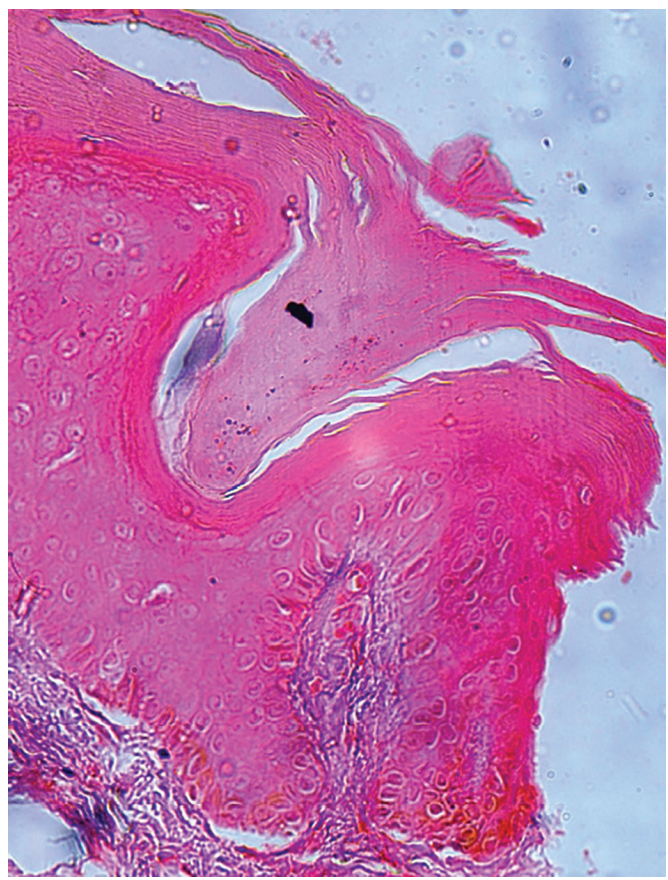

Figure 2 | Histology of the lesion, showing keratin-filled invaginations with large and vacuolated underlying keratinocytes $(\times 100$, hematoxylin and eosin stain). 


\section{Discussion}

Most porokeratosis cases are sporadic, but at times it can be familial, transmitting as an autosomal dominant form with variable penetrance. Infections, autoimmune, genetic, and inflammatory diseases, malignancies, organ transplantation, drugs, ultraviolet radiation, and trauma are the various known triggers of porokeratosis. The centrifugal spread of individual skin lesions is thought to reflect the migration of abnormal clones of cells (1).

The onset of PM usually occurs in childhood, although it can appear at any age. Lesions of PM start as small, brownish papules that later slowly spread peripherally to form irregular, annular, and verrucous plaques. They can increase in size (up to $20 \mathrm{~cm}$ ), which is known as giant PM. The lesions can occur anywhere on the body, including the face, genitalia, oral mucosa, and even the cornea, but they are mostly found on the extremities, involving the palms and soles. The lesions are asymptomatic, but they can be pruritic, especially when involving body folds (1).

Nail changes secondary to porokeratosis are less common, and if they occur they are mostly seen in linear form. They include splitting, longitudinal ridging, nail dystrophy, and pterygium unguis formation (2-10). Involvement of the nail bed and/or nail matrix by abnormal clonal keratinocytes causes onychodystrophy (2). After shedding of the nail plate, the nail bed shows warty debris, which was described as "hyperkeratosis eccentric atrophicans" by Respighi (11). Itin has reported a family with porokeratosis plantaris, palmaris et disseminata with multiple filiform hyperkeratoses and nail dystrophy (10). In a study of a patient with generalized PM with buccal lesions, toenail involvement resembled changes of onychomycosis (12). A few authors have observed shortening, distal digital narrowing, bone resorption, flexion contracture, and mutilation with loss of the digits involved above PM lesions and linear porokeratosis $(2,6,13)$. Hence, patients with PM and linear porokeratosis, especially when involving the extremities, should undergo radiographic evaluation.

Up to $7.5 \%$ cases of porokeratosis may undergo malignant transformation into Bowen's disease, squamous cell carcinoma, and rarely basal cell carcinoma. Longstanding, large porokeratosis lesions, especially those located over limbs, and linear porokeratosis lesions are more prone to malignant degeneration. Loss of heterozygosity may explain the susceptibility of these lesions to malignant changes (14).

Histopathology of porokeratosis is diagnostic. Hyperkeratotic stratum corneum with a column of poorly staining parakeratotic stratum corneum cells (i.e., cornoid lamella) is a characteristic finding in the histopathology of PM, whereas it can be less conspicuous in other forms of porokeratosis. The granular layer is absent beneath the parakeratotic column. The underlying keratinocytes are large and vacuolated (1). Immunosuppression-associated porokeratosis does not show these specific microscopic findings (14).

Treatment of porokeratosis varies according to the type and extent of involvement. Topical therapies such as keratolytics, retinoids, vitamin D derivatives, 5 -flurouracil ointment, imiquimod cream, diclofenac gel, dermabrasion, cryotherapy, photodynamic therapy, carbon dioxide laser, excision, and skin grafting have been used alone or in combination, whereas systemic retinoids are useful in generalized porokeratosis cases. However, none of them have shown promising efficacy and long-term remission (1, $2,14)$. There is no clear consensus on treatment, especially when nail dystrophy is associated with porokeratosis.

Porokeratosis involving distal extremities can lead to onychodystrophy and in some cases underlying bony involvement as well, causing deformity and disability in a patient. Clinicians should be aware of such presentations so that early measures against PM can halt the disease process and any resulting morbidities as well.

\section{References}

1. Oji V, Metze D, Traupe H. Inherited disorders of cornification. In: Griffiths C, Barker J, Bleiker T, Chalmers R, Creamer D, editors. Rook's textbook of dermatology. 9th ed. Chichester, UK: Wiley Blackwell; 2016: Chapter 65; p. 67-68.

2. Kohara Y, Takeo T, Oshima Y, Akita Y, Tamada Y, Watanabe D. Linear porokeratosis with nail dystrophy. Eur J Dermatol. 2011;21:625-6.

3. Kono M, Yokoyama N, Ogawa Y, Takama H, Sugiura K, Akiyama M. Unilateral generalized linear porokeratosis with nail dystrophy. J Dermatol. 2016;43:286-7.

4. Chen $\mathrm{HH}$, Liao $\mathrm{YH}$. Onychodystrophy in congenital linear porokeratosis. Br J Dermatol. 2002;147:1272-3.

5. Robati RM, Roodsari MR, Ayatollahi A, Hejazi S. Facial and bilateral acral porokeratosis with nail dystrophy: a case report. Dermatol Online J. 2011;17:5.

6. Singh N, Chandrashekar L, Kumar N, Kar R, Thappa DM. Classic porokeratosis of Mibelli. Indian Dermatol Online J. 2014;5:130-1.

7. Karthikeyan K, Thappa DM, Udayashankar C. Porokeratosis of Mibelli with nail dystrophy. J Dermatol. 2003;30:420-2.
8. Ouédraogo NA, Ouédraogo SM, Tapsoba GP, Andonaba JB, Niamba P, Traoré A Coexistence of porokeratosis of Mibelli with nail dystrophy to disseminated superficial porokeratosis. Int J Case Rep Images. 2016;7:787-90.

9. Kim DS, Roh MR, Lee JH, Lee KH. Pterygium unguis formation in porokeratosis of Mibelli. Br J Dermatol. 2007;156:1384-5.

10. Itin PH. Porokeratosis plantaris, palmaris et disseminata mit multiplen filiformen Hyperkeratosen und Nageldystrophie. Hautarzt. 1995;46:869-72.

11. Respighi E. Di une ipercheratosi non ancora descritta. G It Dermatol Venereol. 1893;28:356.

12. Kobayasi T. Generalized porokeratosis of Mibelli with lesions of buccal mucosa and of the nails. Jap J Derm Urol. 1934;36:439-42.

13. Handjani F, Shahbaz S, Aslani FS, Gheisari F, Mozaffarian K, Kasraee B. Porokeratosis of Mibelli with mutilation: a case report. Cutis. 2010;86:77-80.

14. Kanitakis J. Porokeratoses: an update of clinical, aetiopathogenic and therapeutic features. Eur J Dermatol. 2014; 24:533-44. 